\title{
Mechanical Characteristics of Reinforced and Three-Layer Shells Based on Metamaterials, Taking into Account Operational Damage
}

\author{
E. V. Lomakin ${ }^{a, b}$, S. A. Yurgenson ${ }^{b, *}$, B. N. Fedulov ${ }^{c, * *}$, and A. N. Fedorenko ${ }^{c}$ \\ ${ }^{a}$ Lomonosov Moscow State University, Moscow, 119991 Russia \\ ${ }^{b}$ Moscow Aviation Institute (National Research University), Moscow, 125080 Russia \\ ${ }^{c}$ Skolkovo Institute of Science and Technology (Skoltech), Moscow, 121205 Russia \\ *e-mail:sjurg@yandex.ru \\ **e-mail:fedulov.b@mail.ru
}

Received March 8, 2021; revised March 19, 2021; accepted March 22, 2021

\begin{abstract}
The conventional design in aeronautical engineering is reinforced shell, which for most structures is a thin shell with a stringer set. This article compares the behavior of a conventional reinforced shell and a spaced shell metamaterial structure with a reinforced set using the example of a main airplane pressure bulkhead. The evaluation criterion is to ensure the required level of residual strength when the limiting state is reached.
\end{abstract}

Keywords: spherical shell, crack resistance, crack propagation model, reinforced shells

DOI: $10.3103 /$ S0025654421060091

\section{INTRODUCTION}

Optimization of the structure of aviation and space technology is one of the main areas of work during design. The aim of optimization is traditionally the weight of the structure, another important parameter is usually the cost of manufacturing, but the requirements for operating costs and maintainability are also an important factor. The factors of operational manufacturability can also act as the main criterion, since the total cost of operating costs and the frequency of maintenance of aviation equipment can significantly exceed the attractiveness of its initial cost.

One of the elements of operational manufacturability is the survivability of the structure, i.e. the ability of a structure to perform tasks in the presence of damage. For aircraft structures, the main requirements for regulated damage are set out in [1]. Such damage includes:

- destruction of one of the elements during multidirectional load transfer;

- cracks in the structure, including the destruction of stringers, spar belts and other elements;

- destruction of one of the joint elements;

- various damage to structures made of composite materials;

- corrosion and shock damage.

Such damages must be taken into account in the design of the structure, when determining the residual strength and the frequency of inspections of the structure or the local place where this damage can occur. To ensure the safe operation of the aircraft, it is necessary to formulate an inspection program that determines the frequency of inspections, the conditions for their conduct and the means of control. The frequency of inspections is determined based on an assessment of the growth of damage from the minimum reliable detectable size to the limit.

For structures made of metallic materials, visual inspection means are the primary means of control, since in many cases the development of damage starts from the surface. For composite structures, the development of damage often begins inside the material, which leads to the need to use a large number of instrumental control methods and significantly increases the inspection time, and also negatively affects the cost of the structure's life cycle. In addition, the presence of undetectable damage imposes additional 


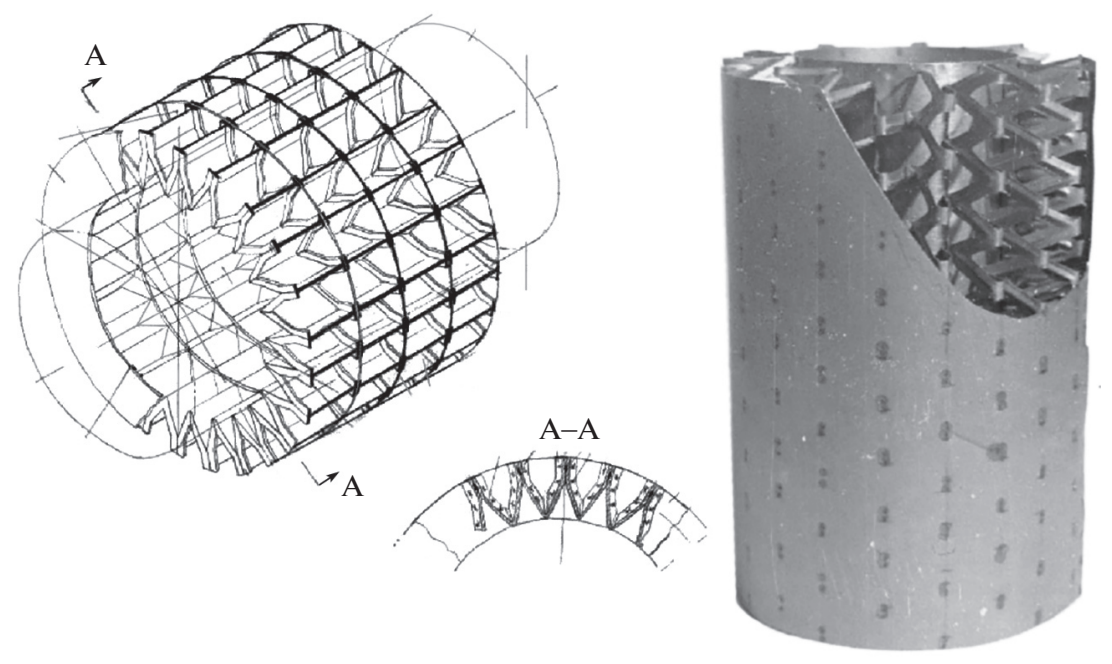

Fig. 1. Structures of A.I. Endogura volume-core fillers.

requirements on the strength of the structure and increases its weight by reducing the allowable stresses to ensure that damage does not grow.

In aviation and space technology, various types of structures and materials are used. The two most common types of structures are reinforced shells and three-layer structures (honeycomb structures). A great contribution to the development of such structures, including spherical shells which are found in rocketry, was made by Feodos'ev [2]. He considered a variety of problems on strength and limiting states, some dynamic problems [3, 4].

Reinforced panels or shells consist of a thin shell and a reinforcing strength set that provide bending stiffness and increase critical stresses (buckling resistance).

Honeycomb or foam-filled designs have a number of disadvantages that limit the scope of their application. These include increased requirements for the parameters of the technological process, the presence of internal defects and their development during operation, the complexity of the design of the docking areas.

To minimize the disadvantages of honeycomb structures, various options with periodic structures are being developed, which would make it possible to reduce the weight of the structure while achieving the required characteristics of strength and rigidity of the structure. One of such solutions is the design of Endogur et al. $[5,6]$ with volume-core filler, pyramidal or wafer filler. The variants of the developed structures are shown in Fig. 1. This structure includes a shell and rigid reinforcements. At the same time, the authors point out the complexity of manufacturing such structures, which are close in structure to human bone tissues, which can be considered one of the factors of optimality of such a structure in terms of load perception.

A volumetric filler was developed with a typical element in the form of a triangular or quadrangular prism, which is made by stamping from a sheet with cut holes of a given shape [7]. Gaynudinov and Aleksandrov developed a variant of the filler, which consists in cutting out zigzag blanks from the sheet with their subsequent connection with the shells through the spikes [8].

These solutions provide for the production of bulk core filler, taking into account the use of traditional technological solutions, but the total labor intensity of the production of such panels is higher than for honeycomb panels. It is necessary to separately mention the structures of Vasiliev, made of composite materials, and representing a mesh structure, which provides quite effective conditions for the operation of reinforcing elements [9].

To solve the problems of optimization of the structure to ensure weight perfection and increase the survivability of the structure, it is proposed to consider structures based on the use of metamaterials. A feature of these structures is the presence of a periodic reinforcing element, in which, in contrast to honeycomb structures, the frequency of the ribs (specific density of the material) can vary, as a result of which the structure can be optimized for strength conditions. At the same time, due to the creation of a sufficiently small elementary cell and the connection of the components with each other, a high survivability of such a structure is achieved - the destruction of one filler element or a separate skin cell does not lead to the destruction of this structure due to the presence of a constructive arrest of crack growth. 
(a)

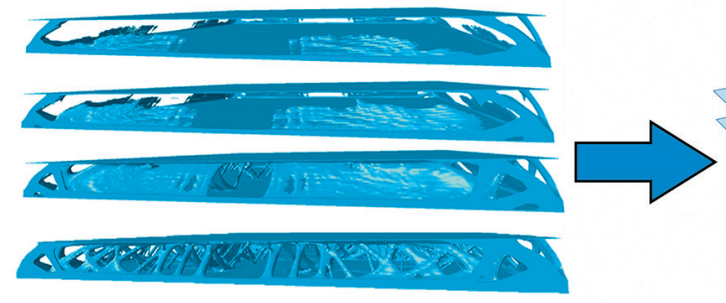

(b)

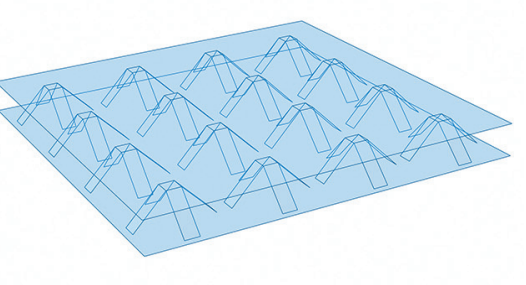

Fig. 2. (a) Stages of material distribution in the optimization process, (b) diagram of the proposed design.

The proposed design consists of two metal sheets connected by a volumetric filler in the form of bent tapes [10-14] (Fig. 2b). The connection of the elements to each other is possible by various types of connections (soldering, welding, mechanical fasteners), while the preferable is the welded connection, which, despite the technologically more complex design and the creation of local stress concentrations, provides a high speed and quality of the connection of the elements. Docking of the panels is also possible with various types of joints, while it is advisable to use mechanical fasteners to simplify the assembly process. The design was formed on the basis of the results of topological optimization (Fig. 2a) and the results of previous work in terms of spatial filler [13, 4]. Figure 2a shows the stages of design optimization during the operation of the topological optimization algorithm. In the formulation of the problem, two independent mutually perpendicular bending moments are considered as force factors.

The advantages of structures based on the principles of metamaterials include:

- good thermal insulation properties;

- uniform reinforcement of shells with filler;

- high coefficient of internal energy absorption;

- the endurance of such structures exceeds the endurance of the panels of stringer structures and increases with a decrease in the shear stiffness of the aggregate;

- long service life due to the absence of rivets causing stress concentration;

- the ability to optimize the strength characteristics by appropriate placement of the filler in the structure.

The disadvantage of this design is the relative complexity of the formation of joining zones in the structure and the presence of internal cavities, which can be centers of corrosion damage in violation of the technology of manufacturing structures.

In contrast to the options proposed above, the design with a filler in the form of stamped or bent metal strips is simpler from a technological point of view, while maintaining the advantages of a bulk filler.

For the perception of concentrated loads, it is possible to compact the periodic structure and locally use thickened metal strips. In the areas of support or docking, a more frequent arrangement of reinforcing elements is used, including with the formation of a tetragonal lattice, similar to that presented above.

In this article, one of the variants of the pressurized bulkhead designs using metamaterials is considered for comparative analysis and assessment of the possibility and efficiency of their use in aircraft structures.

\section{THE TASK OF DESIGNING THE PRESSURE BULKHEAD}

As an example of a structure with a metamaterial, it is proposed to consider the pressure bulkhead of a long-haul aircraft. The pressure bulkhead works to perceive excess pressure in the aircraft cabin. In general, it is a spherical shell with radial ribs (Fig. 3a). In accordance with [1], the regulated damage for such a structure is a crack with a length of $800-1000 \mathrm{~mm}$, oriented in the general case according to the action of maximum stresses.

It is necessary to separately mention the possibility of manufacturing the structure of the pressure bulkhead from composite materials. The solution in Fig. $3 \mathrm{~b}$ provides a weight reduction in comparison with the metal version up to 45\% [15], but, as mentioned above, it loses in terms of technological and operational characteristics.

Within the framework of this work, a simplified design of a pressure bulkhead is considered without taking into account technological and operational hatches for laying communications, since the purpose of this work is a preliminary assessment of the possibility of using structures with metamaterials in aviation technology. The pressure bulkhead was chosen with a diameter of $3 \mathrm{~m}$. For the classical design, the thick- 
(a)

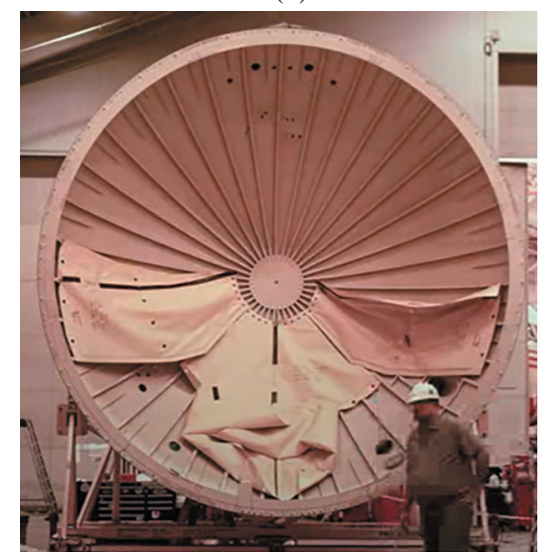

(b)

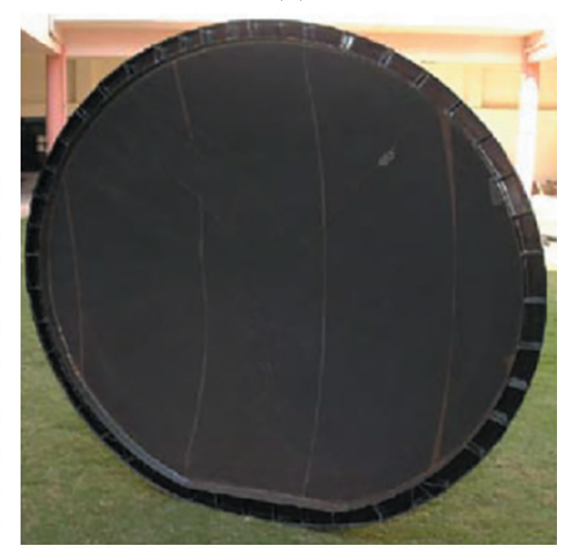

Fig. 3. (a) The metal structure of the pressure bulkhead; (b) compositional structure of the pressure bulkhead.

(a)

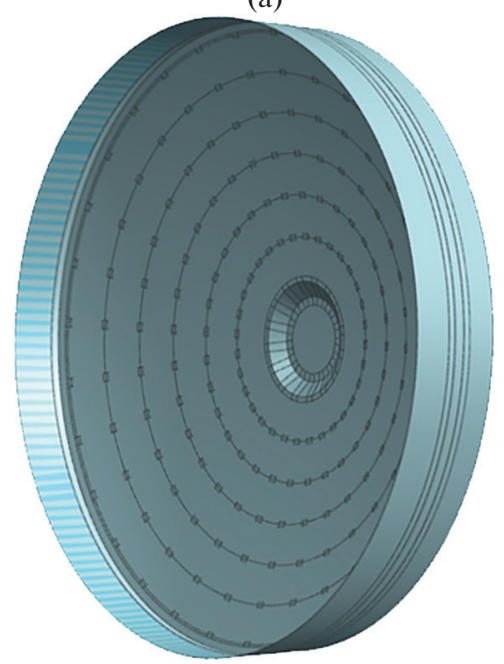

(b)

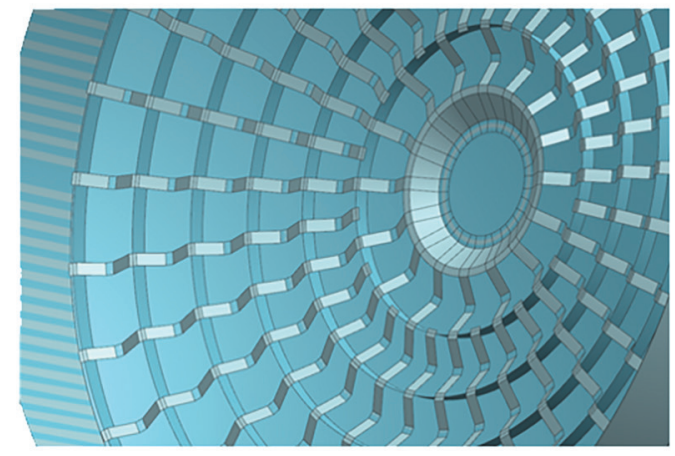

Fig. 4. The structure of the pressure bulkhead using metamaterials: (a) general view, (b) view without one shell.

ness of the shell was taken equal to $1.5 \mathrm{~mm}$. The structure of the pressure bulkhead based on the metamaterial has the same parameters of curvature and diameter, and also provides the same weight of the structure due to the redistribution of weight between the shell and the filler. The thickness of the shells of the proposed design is 1 and $0.5 \mathrm{~mm}$ for the internal and external ones, respectively.

Figure 4 shows the design of the proposed pressure bulkhead made of metamaterials with periodic cells made of sheet aluminum with subsequent through laser welding. This structure is typical and can be optimized taking into account the existing loads from overpressure and concentrated loads ( for example, in the case of joints of keel attachment points to this frame).

The operating pressure is applied as a design load to assess the residual strength of the structure after applying the regulated damage as a crack. In accordance with the requirements of AP-25 p. 25.365 (a) [1], the load from a pressure drop of $0.65 \mathrm{~atm}\left(65861 \mathrm{~N} / \mathrm{m}^{2}\right)$ is considered.

\section{SIMULATION OF THE PRESSURE BULKHEAD LOADING}

The modeling was carried out on the basis of the finite element method. Elements of the shell type with a reduced degree of integration were used. The models used the constitutive relations of the theory of elasticity for isotropic materials, taking into account geometric nonlinearity. The mechanical properties used in the numerical simulation and analysis of aluminum shells are given in Table 1. 
(a)

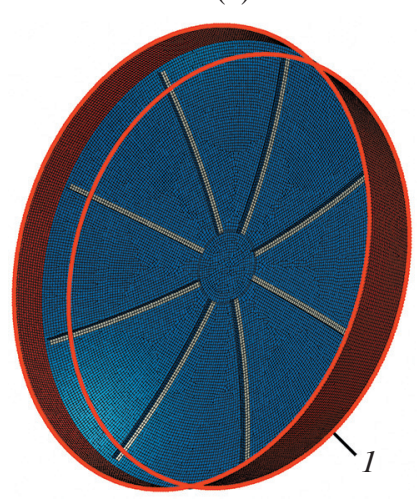

(b)

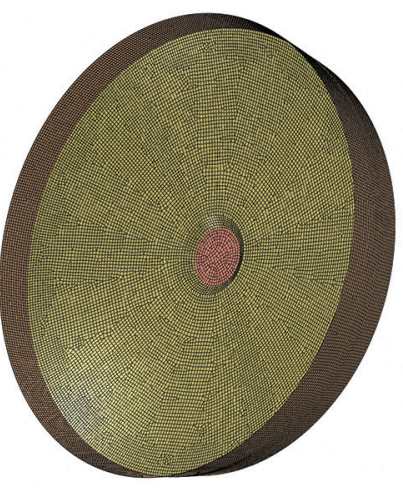

(c)

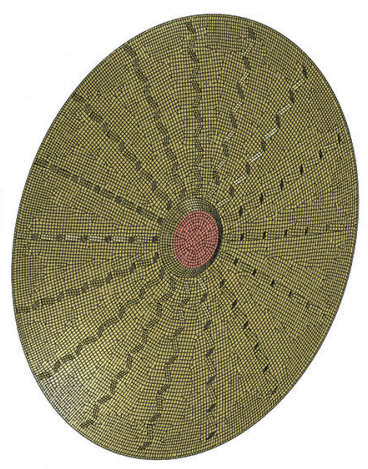

Fig. 5. General view of the numerical grid: (a) classical configuration ( 1 -nodes fixed in the calculation), (b) proposed configuration, (c) proposed configuration (view of the interior)

(a)

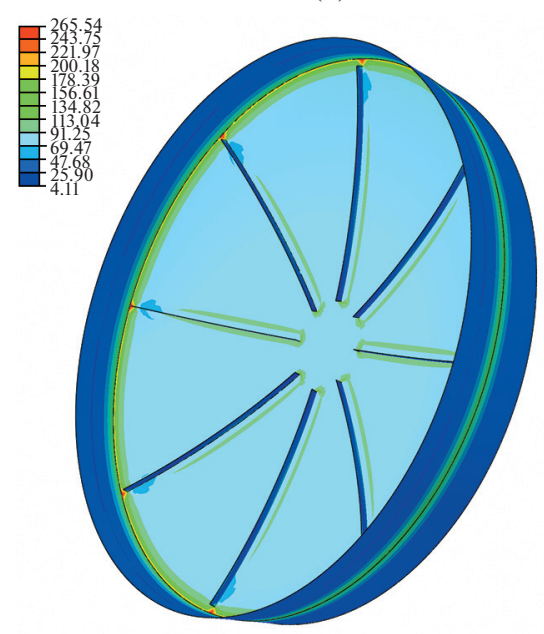

(b)

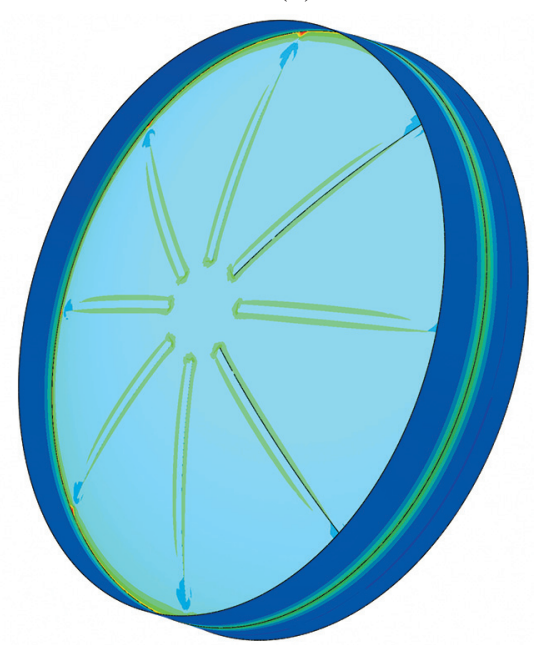

Fig. 6. The maximum thickness value of equivalent von Mises stresses (MPa) for the classical configuration: (a) internal view, (b) external view.

Figure 5 shows a general view of the numerical grids used. The characteristic face size of the element is $20 \mathrm{~mm}$. The number of elements in the model for the classic configuration is 31401, and 43910 for the model with the proposed reinforcement. Fig.5a shows a numerical grid for a classical pressure bulkhead configuration, where highlighted lines indicate nodes that are anchored in all degrees of freedom. The pressure was applied directly to the entire inner surface of the model.

Figure 6 shows the results of stress analysis for a classical configuration based on stringer reinforcements. The maximum thickness value of equivalent von Mises stresses (MPa) is used as the color fill.

Figure 7 shows the results of calculating the stresses for the proposed configuration of the force spherical shell.

Next step is to compare the reliability of the structures. Let us consider the case required by aviation regulations - the presence of a radial crack with a length of 800 to $1000 \mathrm{~mm}$ [1]. In the calculations, it is assumed that in the case of the classical reinforcement scheme, the tightness is broken, but the pressure difference is maintained forcibly. In the second case, we assume that the pressure is redistributed from the first shell to the outer one. Figure 8a depicts the results of a classical configuration based on stringer reinforcements with a radial fracture present. Figures $8 \mathrm{a}$ and $8 \mathrm{~b}$ show the proposed shape of the reinforcements, using the maximum thickness value of von Mises stresses (MPa) as the color fill. It can be seen that in the classical design the stresses are localized in the sector with a crack, while in the proposed design the stresses are distributed almost evenly. 
(a)

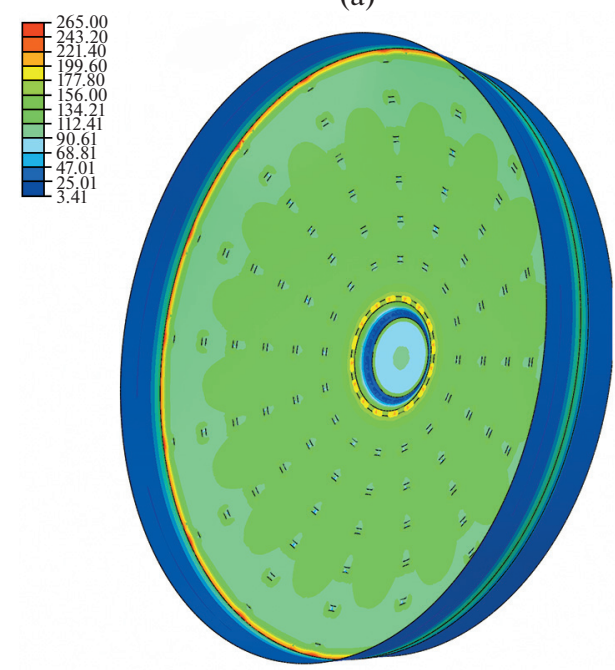

(b)

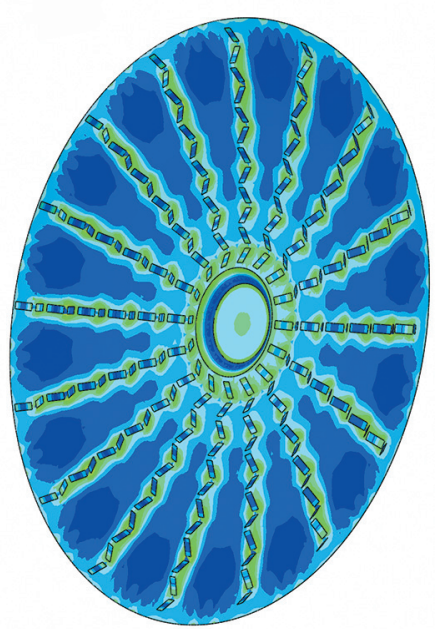

Fig. 7. The maximum thickness value of equivalent von Mises stresses (MPa) for the proposed configuration: (a) internal view, (b) external side of the shell.

(a)

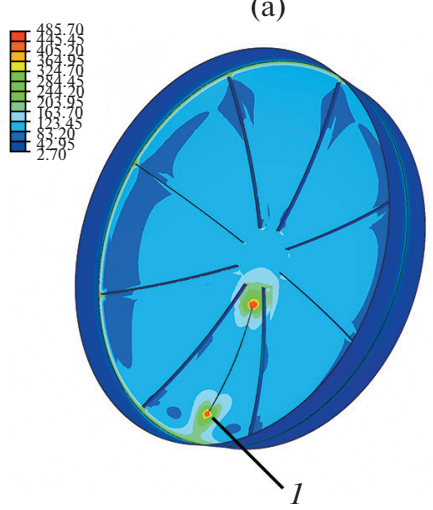

(b)

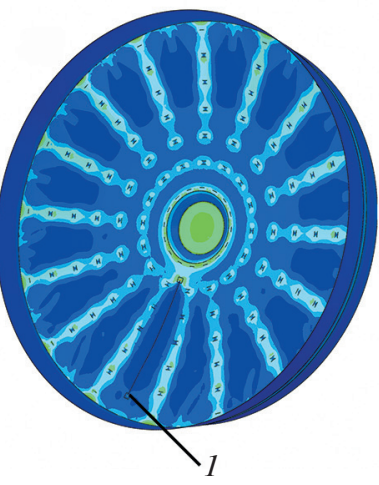

(c)

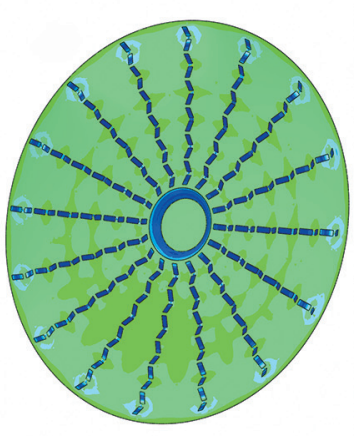

Fig. 8. The result of the calculation in the presence of a crack; the maximum thickness value of equivalent von Mises stresses $(\mathrm{MPa})$ was used in the fill, 1 - crack; (a) classical configuration, (b) proposed configuration, (c) proposed configuration, view of the inner part.

Stresses in regular zones do not exceed the yield point, but to assess the integrity of the structure, it is also necessary to analyze the conditions for crack growth based on the critical value of the stress intensity factor given in Table. 1 . In this article, an approach based on calculating the contour integrals and determining on their basis the values of the stress intensity factors [17-21] was used. The $K_{\mathrm{II}}$ and $K_{\mathrm{III}}$ values turned out to be several orders of magnitude less than the limiting values, which is a characteristic result for thin-walled aircraft structures. The values of the stress intensity factor for normal crack opening $\mathrm{K}_{\mathrm{I}}$, referred to the critical value $K_{\mathrm{c}}$ (Table 1), are shown in Fig. 9. It can be seen that in the classical design the

Table 1. Mechanical properties of aluminum [16]

\begin{tabular}{l|c}
\hline Young's modulus (MPa) & 71000 \\
Poisson's ratio & 0.3 \\
Yield strength $(\mathrm{MPa})$ & 450 \\
Critical stress intensity factor $K_{\mathrm{c}}, \mathrm{MPa} \mathrm{m}^{1 / 2}$ & 37.9 \\
\hline
\end{tabular}




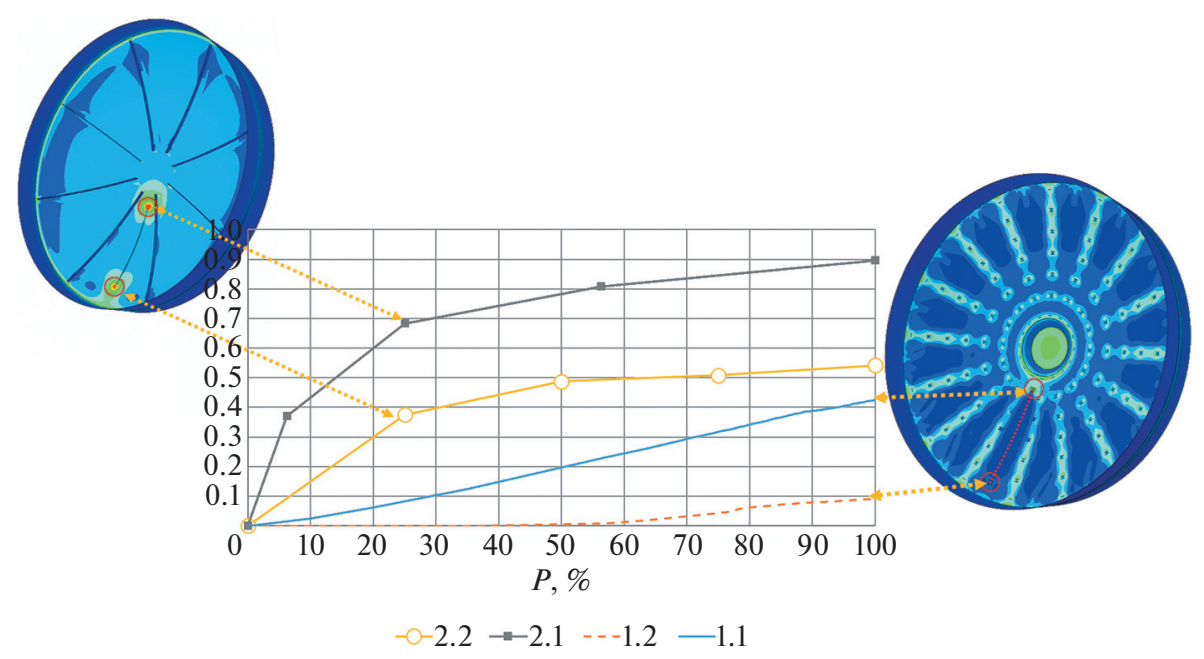

Fig. 9. The result of calculating the stress intensity factors for the classical configuration and for the proposed design: 1.1external crack tip of classical design, 1.2-internal crack tip of classical design, 2.1 -xternal crack tip of the proposed design; 2.2 -internal crack tip of the proposed design; $P(\%)$ - percentage of the applied pressure at the request of $A P-25$ [1] $(0.65 \mathrm{~atm})$.

crack resistance limit is practically reached, while for the proposed design the values of the stress intensity factors are several times lower for both defect tips.

\section{CONCLUSIONS}

This article discusses design options for a sealed bulkhead with regulated damage to assess the possibility of using metamaterial from bent aluminum sheets with outer skin in aircraft structures. The analysis of crack growth for conventional and proposed structures is carried out. With the same mass of the two variants of the pressure bulkhead design, in the proposed variant it was possible to provide a significantly higher structure survivability, estimated on the basis of several times lower values of the stress intensity factors at the crack tips, which are significantly lower than the values obtained for the conventional design.

Based on the results of the studies carried out, it can be concluded that the elements of aircraft structures obtained using stamping, laser welding and other techniques for creating metamaterials can be effectively used in aircraft construction. The presence of two shells and a periodic structure as a filler at the same time ensures high survivability of such structures.

\section{FUNDING}

This research was supported by the Russian Science Foundation (grant no. 20-11-20230).

\section{OPEN ACCESS}

This article is licensed under a Creative Commons Attribution 4.0 International License, which permits use, sharing, adaptation, distribution and reproduction in any medium or format, as long as you give appropriate credit to the original author(s) and the source, provide a link to the Creative Commons license, and indicate if changes were made. The images or other third party material in this article are included in the article's Creative Commons license, unless indicated otherwise in a credit line to the material. If material is not included in the article's Creative Commons license and your intended use is not permitted by statutory regulation or exceeds the permitted use, you will need to obtain permission directly from the copyright holder. To view a copy of this license, visit http://creativecommons.org/licenses/by/4.0/.

\section{REFERENCES}

1. Method for Determining Compliance with Paragraph 25.571. Interstate Aviation Committee. Aviation Register (IAC, Moscow, 1996) [in Russian].

2. V. I. Feodos'ev, Fundamentals of Rocket Flight Engineering (Nauka, Moscow, 1979) [in Russian]. 
3. V. I. Feodos'ev, "To the calculation of corrugated boxes (bellows)," Inzh. Sborn. AN SSSR 4 (1), 137-149 (1947).

4. V. I. Feodos'ev, "On a method of solution of the nonlinear problems of stability of deformable systems," J. Appl. Math. Mech. 27 (2), 392-404 (1963). https://doi.org/10.1016/0021-8928(63)90008-X

5. A. I. Endogyr, S. M. Zhukob, and A. F. Kolganov, Design of Honeycomd Structures with a Volume-Rod Filler: Methods of Synthesis of Modern Aircraft (MAI, Moscow, 1984) [in Russian].

6. A. I. Endogyr, Aircraft Design. Designing Glider Units (MAI-Print, Moscow, 2012) [in Russian].

7. H. N. G. Wadley, N. A. Fleck, and A. G. Evans, "Fabrication and structural performance of periodic cellular metal sandwich structures," Compos. Sci. Technol. 63 (16), 2331-2343 (2003). https://doi.org/10.1016/S0266-3538(03)00266-5

8. V. G. Gainutdinov, S. M. Musavi Safavi, and I. N. Abdullin, "Failure conditions of pyramidal and tetrahedral truss core materials," Vestn. Kazanskogo Gos. Tekh. Uni. A. N. Tupoleva 71 (2), 11-15 (2015).

9. V. V. Vasiliev, V. A. Barynin, and A. F. Rasin, "Anisogrid lattice structures-survey of development and application," Compos. Struct. 54 (2-3), 361-370 (2001). https://doi.org/10.1016/S0263-8223(01)00111-8

10. V. S. Deshpande and N. A. Fleck, "Collapse of truss core sandwich beams in 3-point bending," Int. J. Solids Struct. 38 (36-37), 6275-6305 (2001).

11. H. N. G. Wadley, "Multifunctional periodic cellular metals," Phil. Trans. Royal Soc. A: Math. Phys. Eng. Sci. 364 (1838), 31-68 (2006).

12. G. W. Kooistra and H. N. G. Wadley, "Lattice truss structures from expanded metal sheet," Mater. Des. 28 (2), 507-514 (2007).

13. B. N. Fedulov, A. N.Fedorenko, S. A. Jurgenson, et al., "Construction plate enforced by metamaterial elements," Procedia Structural Integrity. 28, 155-161 (2020). https://doi.org/10.1016/j.prostr.2020.10.020

14. S. A. Yurgenson, E.V. Lomakin, B.N. Fedulov, and A.N. Fedorenko, "Structural elements based on the metamaterials,” Vestn. PNIPU Mekh., No. 4, 211-219 (2020). https://doi.org/10.15593/perm.mech/2020.4.18

15. S. Venkatesh, et al., "Design of a composite rear pressure bulkhead for a light transport aircraft," in the Proceedings of ISAMPE National Conference on Composite Structures, December 2009, Hiruvananthapuram, India (ISAMPE, 2009).

16. S. I. Kishkina and I. N. Fridlander, Aviation Materials, Ed. by R. E. Shalin, Vol. 4: Aluminum and Beryllium Alloys, Part. 1: Wrought Aluminum Alloys and Beryllium-Based Alloys, Book 1 (ONTI, Moscow,1082) [in Russian].

17. Y. Lei, "J-integral evaluation for cases involving non-proportional stressing," Eng. Fract. Mech. 72 (4), 577$596(2005)$. https://doi.org/10.1016/j.engfracmech.2004.04.003

18. G. P. Cherepanov, "Crack propagation in continuous media," J. Appl. Math. Mech. 31 (3), 503-512 (1967). https://doi.org/10.1016/0021-8928(67)90034-2

19. J. R. Rice, "A path independent integral and the approximate analysis of strain concentration by notches and cracks,” ASME J. Appl. Mech. 35 (4), 379-386 (1968).

20. V. D. Klyushnikov, Physico-Mathematical Foundations of Strength and Plasticity (MGU, Moscow, 1994) [in Russian].

21. N. F. Morozov, Mathematical Problems of the Theory of Cracks (Nauka, Moscow, 1984) [in Russian].

Translated by M. K. Katuev 